\title{
Un panorama de la jeune recherche en sciences humaines et sociales sur l'eau. Retour sur trois éditions des «Doctoriales en sciences sociales de l'eau »
}

\author{
Sylvain Barone ${ }^{1}$, David Blanchon ${ }^{2, *}$ et François Destandau ${ }^{3}$ \\ 1 Science politique, Irstea, UMR G-eau, Montpellier, France \\ ${ }^{2}$ Géographie, Université Paris Nanterre, UMR7218 Lavue, Nanterre, France et UMI Iglobes, Tucson, États-Unis \\ 3 Économie, Engees, UMR Geste, Strasbourg, France
}

\begin{abstract}
Résumé - Entre 2014 et 2017, trois Doctoriales ont été l'occasion pour 84 doctorants en sciences humaines et sociales de présenter leur travail de thèse sur un objet commun : l'eau. À l'aide de ce matériau et d'une courte enquête complémentaire réalisée en 2017, cet article vise à analyser ce qu'est aujourd'hui la jeune recherche en sciences humaines et sociales sur l'eau. Principalement inscrites en géographie et en économie, ces thèses regroupent une douzaine de disciplines. Une grande variété d'approches et de méthodes sont observées, avec toutefois des spécificités disciplinaires : approche normative ou constructiviste, références plutôt anglo-saxonnes ou francophones, etc. Des tendances plus générales ont également pu être identifiées, comme une pratique répandue de la pluridisciplinarité et le souhait majoritaire, pour ces jeunes chercheuses et chercheurs, de rester dans le circuit académique après le doctorat.
\end{abstract}

Mots-clés : sciences humaines et sociales / eau / doctorants / jeunes chercheurs

\begin{abstract}
An overview of junior researchers and water studies in France through the experience of three “Doctoriales en sciences sociales de l'eau”. Between 2014 and 2017, during three "Doctoriales" sessions, 84 young $\mathrm{PhD}$ candidates in humanities and social sciences were given the opportunity to present their work. Based on this experience, and a short survey done in 2017 by the authors, this paper aims to analyze the state of "young" research on water in human and social sciences. Even if most of the papers presented during the "Doctoriales" relate to geography and economy, overall 12 disciplinary fields were represented. A great variety of approaches and methods were also noted with, however, disciplinary specificities concerning a normative or constructivist approach, references mainly to Anglo-Saxon or French literature, and so on. General trends have also been identified, such as a widespread multidisciplinary approaches and, for most young researchers, the wish to remain in academia after their $\mathrm{PhD}$.
\end{abstract}

Keywords: water studies / humanities and social sciences / $\mathrm{PhD}$ candidates / junior researchers

\section{Les Doctoriales comme laboratoire d'étude de la jeune recherche en SHS de l'eau}

Les sciences du vivant et les sciences de l'ingénieur concentrent la grande majorité des recherches dans le domaine de l'eau. Les approches de sciences humaines et sociales sur cet objet n'en sont pas moins d'une extrême variété et d'une immense richesse, et sont d'ailleurs de plus en plus sollicitées dans les appels à projets de

\footnotetext{
*Auteur correspondant : dblanchon@gmail.com
}

recherche. Il semble dès lors intéressant de tenter de dresser un état des forces en présence. Ce court texte ne vise pas à brosser un panorama exhaustif des sciences humaines et sociales (SHS) de l'eau mais à voir ce que trois éditions de «Doctoriales en sciences sociales de l'eau ${ }^{1} »$ peuvent nous apprendre sur l'état actuel de la recherche dans ce champ.

\footnotetext{
${ }^{1}$ Les auteurs, François Destandau, Sylvain Barone et David Blanchon, membres du comité scientifique des Doctoriales, ont respectivement présidé les comités d'organisation des Doctoriales de Strasbourg, Montpellier et Nanterre.
} 
Les Doctoriales francophones en sciences sociales de l'eau sont nées du constat qu'en dehors de quelques grands pôles, la recherche en SHS sur l'objet eau est très disséminée en France et dans le monde francophone. Beaucoup de doctorants travaillent seuls dans leur laboratoire sur cet objet avec des approches très diverses. Il apparaissait dès lors compliqué de quantifier et d'identifier les thèses en cours sur ces questions. L'idée simple de départ était ainsi de réunir tous ces jeunes chercheurs. L'enthousiasme suscité par la première édition strasbourgeoise de 2014 a, d'une certaine manière, confirmé que cet évènement manquait. La décision fut donc prise de poursuivre l'expérience à un rythme d'une édition tous les ans et demi, soit la moitié d'une thèse, permettant aux doctorants de présenter leurs travaux en début et en fin de thèse. Des éditions estivales et hivernales se succèdent. Un comité scientifique a été également constitué pour assurer cette pérennité. Il est aujourd'hui constitué de 27 chercheurs seniors de Bordeaux, Limoges, Lyon, Montpellier, Nanterre, Paris, Rennes, Strasbourg, Toulouse, en économie, géographie, sociologie, science politique, ethnologie et anthropologie.

L'objet de ce texte est de revenir sur les trois premières éditions des Doctoriales afin de dresser un bilan des recherches menées par la jeune recherche dans le domaine de l'eau et de dessiner les fronts de recherche actuels et les centres d'intérêt des futurs chercheurs et chercheuses en sciences humaines et sociales au sens large (du droit aux sciences économiques en passant par la géographie, la sociologie, l'histoire, l'anthropologie...). Nous nous appuyons pour cela sur les fiches de renseignements que nous avons demandé à chacun et chacune de remplir au moment de l'inscription aux Doctoriales, sur les informations recueillies avant chaque édition des Doctoriales par le comité scientifique sur le devenir des anciens participants, et sur les résultats d'un questionnaire $a d$ hoc qui a été envoyé à l'ensemble des participantes et participants afin de recueillir des informations supplémentaires (sur le financement de thèse, l'inscription disciplinaire et la pratique de l'interdisciplinarité, les positions et perspectives professionnelles...2).

\section{Trois éditions des Doctoriales}

\section{Première édition : Strasbourg, 4-5 décembre 2014}

Les premières Doctoriales en sciences sociales de l'eau ont été organisées par l'UMR Geste les 4 et

\footnotetext{
${ }^{2}$ Un lien vers le questionnaire a été envoyé par e-mail. Nous avons obtenu 40 réponses, soit environ la moitié de l'ensemble des participantes et participants aux Doctoriales. Nous remercions vivement Mathilde Fautras, qui s'est occupée de cette enquête complémentaire.
}

5 décembre 2014 à l'École nationale du génie de l'eau et de l'environnement de Strasbourg (Engees). Elles ont rassemblé 61 personnes, dont 25 jeunes chercheurs et chercheuses venus de France, de Suisse et de Belgique pour présenter leur travail de thèse, 10 chercheuses et chercheurs confirmés sollicités pour discuter et présider les sessions, un "grand témoin» (Jean Verdier), le président de la Société hydrotechnique de France (SHF), et le lauréat du prix de thèse SHF. Cet évènement a reçu le soutien financier et logistique de l'Engees, de l'Irstea, de l'Agence de l'eau Rhin-Meuse, de la SHF, de l'École doctorale Augustin-Cournot, de la Casden, de la ville et de la communauté urbaine de Strasbourg, et enfin de l'Association française pour l'eau, l'irrigation et le drainage (AFEID).

Les thématiques des sessions s'intitulaient: «Statut et représentation des eaux», «Services d'eau», «Eau agricole», "Changements des politiques territorialisées », «Démocratie de l'eau», «Inondations », «Accès à l'eau des villes du Sud», «Continuité et restauration des cours d'eau ».

Une présentation des possibilités d'emploi pour les docteurs en SHS par une consultante de l'Association Bernard Gregory a également eu lieu. Joël Cabalion, le lauréat du prix de thèse sur l'eau en sciences sociales de la SHF (prix Pierre Massé) 2014, a également présenté son travail sur les mobilisations paysannes lors de la construction du grand barrage Gosikhurd en Inde centrale ${ }^{3}$.

\section{Deuxième édition : Montpellier, 16-17 juin 2016}

La deuxième édition a été organisée par l'Institut montpelliérain de l'eau et de l'environnement (IM2E) les 16 et 17 juin 2016. Elle a rassemblé à Montpellier 89 personnes, dont 48 jeunes chercheuses et chercheurs venus de 6 régions françaises, de Suisse, de Belgique, de Tunisie, de Haïti et du Québec pour présenter leur travail de thèse, 16 chercheuses et chercheurs confirmés venus encadrer les sessions, le vice-président de Montpellier Méditerranée Métropole (René Revol), un "grand témoin» scientifique (Alice Ingold), ainsi que la lauréate du prix de thèse Pierre Massé 2015 de la SHF (Alix Levain). Cet évènement a reçu le soutien financier et logistique de l'IM2E, de l'Institut national de recherche en sciences et technologies pour l'environnement et l'agriculture (Irstea), de l'Université PaulValéry-Montpellier 3, de l'École doctorale ABIES (Agriculture, alimentation, biologie, environnement,

\footnotetext{
${ }^{3}$ Cabalion J., 2013. Des existences paysannes au fil de l'eau: le grand barrage Gosikhurd et les déplacements de population au Vidarbha, Inde centrale. Thèse de doctorat en sociologie, Paris, École des hautes études en sciences sociales.
} 
santé), de Montpellier Méditerranée Métropole, de la SHF et de Montpellier SupAgro.

Les thématiques des sessions s'intitulaient : «Participation, délibération », "Gouvernance territoriale », "Accès, droits», «Représentations, attachements», «Demande et allocation», «Indicateurs», «Pouvoir et mise en politique », «Risques », " Aménagements », «Réformes, action collective, métiers ». Certains doctorants ont présenté leur thèse en session posters. Le poster était accompagné d'une présentation en 180 secondes. Alix Levain a exposé son travail de thèse en ethnologie intitulé Vivre avec l'algue verte: médiations, épreuves et signes ${ }^{4}$. Cette édition a été valorisée par un ouvrage collectif réunissant une partie des meilleures contributions 5 .

\section{Troisième édition : Nanterre, 4-6 décembre 2017}

Les troisièmes Doctoriales ont eu lieu à l'Université Paris Nanterre les 4, 5 et 6 décembre 2017. Ces journées ont été organisées et soutenues par le Réseau d'études et d'échanges en sciences sociales sur l'eau (Rés-EAUx ${ }^{6}$ ), l'UMR Lavue, l'équipe Mosaïques, la Maison Archéologie et Ethnologie René-Ginouvès (MAE), et l'Institut universitaire de France (IUF). Une centaine de personnes ont participé, dont 35 jeunes chercheuses et chercheurs venus d'̂̂le-de-France, de Montpellier, de Strasbourg, de Lyon, de Toulouse, d'Orléans, de Bordeaux, de Valenciennes, de Rennes, mais également de Haïti, de Suisse, du Niger, du Bénin, des États-Unis et du Canada.

Cette édition a été organisée autour de six panels: « Gouverner et gérer l'eau », «Parler de l'eau, faire parler l'eau: archives, récits, discours», "Prix et valeurs de l'eau », « Construire et détruire des barrages », « Se battre pour l'eau, se battre avec l'eau: conflits et inégalités d'accès l'eau», et enfin "Contrôler et sécuriser les ressources en eau». Durant ces trois jours, d'autres évènements ont été programmés, comme la projection du film documentaire La Dordogne vue par ses habitants, réalisé par Jamie Linton et Émilie Crémin, qui est un recueil de témoignages de riverains confrontés à la modification de la Dordogne au cours du temps par la construction de barrages; la rencontre entre les doctorants et des professionnels sous la forme d'un speed networking (premier échange de 2 minutes entre jeune chercheur ou chercheuse et professionnel où chacun se présente, suivi d'un temps de discussion libre); une exposition photos sur le thème des techniques

\footnotetext{
${ }^{4}$ Levain A., 2014. Vivre avec l'algue verte: médiations, épreuves et signes. Thèse de doctorat en anthropologie de l'environnement, Paris, Muséum national d'histoire naturelle.

${ }^{5}$ Barone S., Barbier R., Destandau F., Garin P. (Eds), 2018. Gouvernance de l'eau: un mouvement de réforme perpétuelle?, Paris, L'Harmattan.

${ }^{6} \mathrm{https}: / /$ reseaux.parisnanterre.fr/
}

d'accès à l'eau; la présentation d'un ouvrage ${ }^{7}$ dans le cadre de l'exposition Bibli-Eau «L'apport des sciences sociales aux études environnementales », ainsi que la présentation de la thèse en économie de Anne-Gaëlle Figureau, lauréate du prix Pierre Massé 2016, intitulée Conception et évaluation d'instruments de gestion de l'eau combinant incitations économiques et préférences sociales ${ }^{8}$.

\section{Que disent les Doctoriales de la jeune recherche en SHS sur l'eau?}

84 doctorantes et doctorants ont participé à une, deux, voire aux trois éditions des Doctoriales. $80 \%$ viennent de France, $20 \%$ effectuent leur thèse hors de nos frontières, dont un peu moins de la moitié sont rattachés à un laboratoire africain : Burkina Faso, Togo, Tunisie, Niger, Bénin. À noter, qu'à chaque Doctoriale, des jeunes chercheurs africains ont dû renoncer à venir par manque de moyens ou parce qu'un visa n'a pu être obtenu. Par ailleurs, les Doctoriales ont accueilli des doctorantes et doctorants venant des États-Unis, du Canada, de Haïti, de Belgique, de Suisse et d'Inde. Pour la France, sans surprise, la moitié est rattachée à un laboratoire d'Île-deFrance ou montpelliérain. Les autres effectuent leur thèse à Lyon, Bordeaux, Strasbourg, Tours, Toulouse, Rennes, Grenoble, Clermont-Ferrand, Limoges, Reims, Pau, Orléans, Valenciennes ou Caen. Conformément à son objectif initial, cet évènement parvient ainsi à rassembler des doctorantes et doctorants des "gros pôles» ou d'autres plus isolés.

La figure 1 illustre la répartition des disciplines représentées lors des trois Doctoriales. Lorsque plusieurs disciplines sont affichées pour un même doctorant (exemple « Aménagement-Sociologie »), chacune compte pour moitié. La catégorie «autres» regroupe les disciplines les moins représentées: urbanisme, sciences de l'environnement, sciences de gestion, archéologie, anthropologie, biologie, génie des procédés, sciences de l'eau. Une partie de ces thèses ne relèvent pas des SHS proprement dites, mais comprennent au moins un volet traitant de problématiques communes aux SHS ; c'est pourquoi nous les avons acceptées. D'autres propositions relevant de l'hydrologie stricto sensu, comme la modélisation des flux d'eau souterraine par exemple, sans aucune mention de leurs modalités d'utilisation, ont été refusées.

\footnotetext{
${ }^{7}$ Haag P., Lemieux, C. (Eds), 2012. Faire des sciences sociales. Critiquer, Paris, Éditions de l'EHESS.

${ }^{8}$ Figureau A.-G., 2015. Conception et évaluation d'instruments de gestion de l'eau combinant incitations économiques et préférences sociales. Thèse de doctorat en sciences économiques, Montpellier, SupAgro.
} 


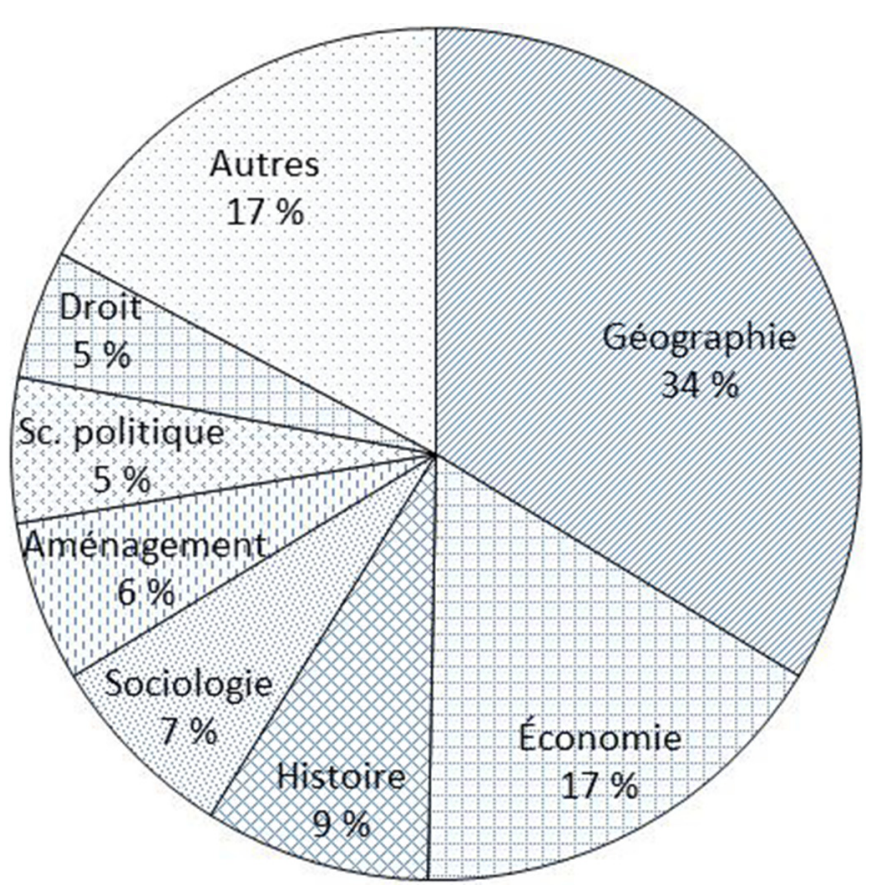

Fig. 1. Répartition des disciplines représentées aux Doctoriales.

La géographie représente le tiers des thèses présentées. C'est surtout lors des Doctoriales de Nanterre que la géographie fut surreprésentée avec la moitié des thèses dans cette discipline. Arrivent ensuite les sciences économiques avec $17 \%$ des thèses. On trouve ensuite entre $5 \%$ et $9 \%$ des thèses en histoire, sociologie, aménagement, science politique et droit. Deux disciplines représentent donc la moitié des contributions, l'économie et la géographie.

Concernant l'économie, une grande partie des thèses traitent de la gestion quantitative de la ressource en eau, et plus particulièrement des eaux d'irrigation, très souvent dans les pays en développement. L'aspect qualitatif est plus rarement abordé. Les autres objets traités sont l'eau dans les services urbains, le risque inondation, l'eau dans la production électrique, ainsi que la question de la continuité écologique. Les questions abordées sont en grande partie liées à l'impact, l'efficacité des politiques publiques ou les modes de gouvernance. Certaines thèses s'intéressent davantage aux comportements des agents soumis à des risques par exemple, d'autres à des indicateurs d'inégalité d'accès à la ressource ou pour mesurer une évolution de la demande. Les branches de l'économie affichées sont très variées: microéconomie, économie rurale, territoriale, institutionnelle, du développement, et ces thèses font également souvent référence à d'autres disciplines: agronomie, psychologie, géographie, hydrologie, aménagement. Les approches peuvent être théoriques (théorie de la décision, théorie des choix collectifs, modèle principal-agent, modèle input-output...) ou empiriques avec des analyses économétriques sur des bases de données ou du traitement statistique d'enquêtes de terrain, parfois complétées d'analyses de discours.

Les bibliographies des papiers présentés lors des Doctoriales indiquent que ce sont les économistes qui mobilisent le plus systématiquement des références internationales. Réciproquement, ils cherchent à publier le plus souvent dans des revues anglosaxonnes. Cela renvoie à une structuration du champ disciplinaire et à une culture scientifique en partie différentes des autres SHS.

En géographie, les enquêtes qualitatives dominent très largement. Et l'on note une quasi-absence d'approches centrées sur les questions hydrologiques, et $a$ fortiori en géomorphologie. Cela est sans doute dû à la dichotomie toujours persistante entre une géographie «physique» qui ne s'identifie pas aux SHS et une géographie « humaine » qui y trouve pleinement sa place. D'autre part, si les questions abordées et les terrains choisis sont très variés, comme en économie, on note une certaine influence de la littérature scientifique anglophone, notamment de la Political Ecology, et secondairement des travaux sur les «communs» à la suite d'Elinor Ostrom.

Certaines SHS (comme la sociologie, la géographie, la science politique) ont longtemps été et restent encore largement résistantes à l'internationalisation (participation à des programmes de recherche et des colloques internationaux, publications, comités éditoriaux, séjours à l'étranger, etc.), pour différentes raisons tenant à la fois, là aussi, à des cultures scientifiques (par exemple une conception de la science peu compatible avec les attentes de certains projets de recherche partenariale jugées trop opérationnelles ou trop normatives) mais également à un fonctionnement organisationnel marqué par l'endorecrutement (français voire microlocal), de lourdes charges d'enseignements, etc. ${ }^{9}$. Ce localisme a longtemps contribué à une insertion limitée dans les débats internationaux. En conséquence, on ne peut que se féliciter de l'ouverture sur le monde anglophone des jeunes chercheuses et chercheurs en SHS sur l'eau, surtout si cela ne se fait pas au prix de l'oubli ou du moins de la marginalisation dans les bibliographies d'auteurs francophones ayant défriché à leur manière de nombreux champs de recherche.

\footnotetext{
${ }^{9}$ Wieviorka M. (avec Moret J.), 2017. Les sciences humaines et sociales françaises à l'échelle de l'Europe et du monde. Rapport au secrétaire d'État à l'Enseignement supérieur et à la Recherche, Paris, Éditions de la Maison des sciences de l'homme.
} 
En termes d'approches théoriques et conceptuelles, toutes disciplines confondues, les travaux d'Ostrom semblent moins omniprésents que dans la littérature sur l'eau en économie (institutionnelle), science politique/ analyse des politiques publiques, mais aussi géographie, sociologie, etc. L'explication par la faible internationalisation des débats est sans doute toujours en partie valable mais, on vient de l'évoquer, devient de moins en moins pertinente. Une autre explication pourrait résider dans le fait que ces approches oscillent entre indifférence et grande méfiance à l'égard de l'État (un État monolithique et d'abord présenté comme un vecteur de domination). Or, en France, la gestion communautaire, par les usagers (celle qui intéresse les courants ostromiens), a été prise en tenaille historiquement entre la propriété privée et, justement, l'État, acteur dont on perçoit rapidement le caractère incontournable et, en même temps, multiface et complexe. Enfin, ces approches ont une portée normative (définir les principes d'une «bonne» gestion de la ressource). Or, nous avons évoqué le rejet d'une grande partie des sciences sociales françaises à l'égard des positionnements normatifs.

De façon générale, dans la plupart des disciplines mobilisées (certes moins en économie et en droit), les dimensions constructivistes et critiques sont très présentes: beaucoup de travaux partent de l'idée que les objets et phénomènes étudiés (le bassin-versant, par exemple) sont des constructions sociales ou politiques. Les paradigmes gestionnaires dominants (la Gestion intégrée des ressources en eaux - GIRE - notamment) sont mis en controverses. La «boîte noire» de certains dispositifs (indicateurs de gestion, par exemple) est ouverte. La naturalité de certains rapports de causalité, comme les effets de la participation, est questionnée. En bref, ces travaux reconstituent l'épaisseur historique, sociale et politique de l'eau, et permettent de faire sortir toujours plus cet objet des sphères techniques et gestionnaires dans lesquelles il a longtemps été cantonné.

Outre les dimensions constructivistes et/ou critiques, un autre aspect notable de la jeune recherche en SHS sur l'eau réside dans l'ouverture disciplinaire. Les participantes et participants aux Doctoriales sont en effet très peu nombreux à être ancrés dans une seule discipline. Une majorité affiche une discipline principale et une ouverture vers une ou plusieurs autres disciplines, et environ un tiers se réclame clairement d'une approche interdisciplinaire. Nouveau «passage obligé» dans le métier de chercheur? Il est vrai que l'interdisciplinarité est perçue positivement dans les projets de recherche internationaux et nationaux. Elle l'est moins, toutefois, au moment des qualifications aux fonctions de maître de conférences et des concours de recrutements à l'Université et au CNRS (la situation étant plus ambivalente dans d'autres établissements de recherche, où la pluri/ interdisciplinarité peut être mieux perçue). Mais les jeunes chercheuses et chercheurs en sont-ils vraiment informés en amont? La croyance dans les vertus de l'interdisciplinarité, y compris de la part des directeurs et membres de comités de thèse, se heurte parfois à un fonctionnement de la recherche qui reste encore, au concret, largement disciplinaire. L'ouverture disciplinaire des jeunes chercheuses et chercheurs en SHS de l'eau est-elle par ailleurs liée à un objet d'étude multidimensionnel, échappant de ce fait sans cesse à celui ou celle qui voudrait l'appréhender à partir d'un unique point de vue disciplinaire, et débouchant assez naturellement sur l'articulation de grilles de lecture, que ce soit à l'intérieur des SHS ou entre SHS et sciences biophysiques, par exemple pour mieux qualifier des interactions homme-milieu? Sans doute également. Mais on ne peut ici qu'inciter à la prudence. Ces données mériteraient en effet d'être comparées à des thèses sur d'autres objets de recherche, dont beaucoup peuvent prétendre au caractère multidimensionnel. Enfin, même si les résultats du sondage ne sont pas forcément significatifs, il semble que la plupart des doctorantes et des doctorants ne sont pas inclus directement dans des programmes de recherches. Cela ne les empêche pas de participer ponctuellement aux activités de programmes nationaux et internationaux.

Au terme de ces trois éditions, plusieurs pistes ont été proposées par les doctorantes et doctorants pour améliorer ces rencontres. L'une d'elles consistait à renforcer le volet «professionnalisant» et les interactions avec les acteurs non académiques. Une telle demande interroge sur la nature des perspectives professionnelles envisagées et pourrait signifier que les carrières dans l'enseignement et la recherche représentent de moins en moins le seul aboutissement de la thèse pour de nombreux jeunes chercheuses et chercheurs, que ce soit par pragmatisme au vu du nombre de postes proposés dans ce secteur, ou par intérêt pour d'autres métiers (coopération internationale, consultance, etc.) où le doctorat en SHS peut constituer un diplôme reconnu-à l'inverse de la fonction publique. Il existe dans le domaine de l'eau des entreprises en recherche de compétences en SHS, qu'un doctorat vient à l'évidence sanctionner: en analyse économique, en animation de démarches ou de contrats, en «acceptabilité sociale» de projets, etc. L'un des objectifs des Doctoriales, sur la durée, est aussi de faire évoluer le point de vue des entreprises potentiellement intéressées par ce type de compétences en les convaincant par exemple davantage de l'intérêt de travaux à portée analytique ou réflexive (et non normative ou immédiatement opérationnelle).

En même temps, il y a là une forme de paradoxe. Il ressort en effet de notre questionnaire qu'une écrasante majorité des participantes et participants aux Doctoriales envisage d'abord un avenir professionnel dans l'enseignement supérieur et/ou la recherche. Celles et ceux qui 
ont soutenu leur thèse s'orientent d'ailleurs de fait en majorité vers ce type de carrière. À ce titre, le potentiel opérationnel valorisable professionnellement lié à l'objet « eau», souvent mis en avant (y compris pendant les Doctoriales), ne semble que marginalement se traduire in fine par une diversification des orientations et des carrières. Ce paradoxe n'en est toutefois peut-être pas un : les doctorantes et doctorants ont désormais bien conscience des difficultés de recrutement dans l'enseignement supérieur et la recherche et connaissent le risque de la stratégie consistant à mettre tous ses œufs dans le même panier. Même si leur objectif premier continue d'être l'enseignement et/ou la recherche, ils jugent vraisemblablement plus réaliste d'avoir plusieurs cordes à leur arc...

Citation de l'article : Barone S., Blanchon D., Destandau F., 2019. Un panorama de la jeune recherche en sciences humaines et sociales sur l'eau. Retour sur trois éditions des «Doctoriales en sciences sociales de l'eau». Nat. Sci. Soc. 27, 3, 336-341. 